Nouvelles perspectives en sciences sociales

\title{
Positionnement des sociologues dans la démarche de modélisation Domino
}

\author{
William's Daré, Christine Fourage et Ibrahima Diop Gaye
}

Volume 2, numéro 2, mars 2007

URI : https://id.erudit.org/iderudit/602461ar

DOI : https://doi.org/10.7202/602461ar

Aller au sommaire du numéro

\section{Éditeur(s)}

Prise de parole

ISSN

1712-8307 (imprimé)

1918-7475 (numérique)

Découvrir la revue

\section{Citer cet article}

Daré, W., Fourage, C. \& Diop Gaye, I. (2007). Positionnement des sociologues dans la démarche de modélisation Domino. Nouvelles perspectives en sciences sociales, 2(2), 103-126. https://doi.org/10.7202/602461ar
Résumé de l'article

Au Sénégal et à La Réunion, gestion décentralisée des ressources naturelles et multiplicité des usages de l'espace amènent les gestionnaires du territoire à penser les complémentarités et compétitions entre secteurs d'activité et niveaux d'organisation. Des outils de représentation des interactions entre sociétés et écosystèmes et de leurs dynamiques existent mais peu sont construits avec les acteurs.

Domino s'appuie sur la modélisation d'accompagnement. Dès ses premières étapes, cette démarche participative implique des chercheurs de disciplines différentes et des acteurs producteurs d'informations et potentiels utilisateurs. Le projet souhaite renforcer les capacités des acteurs pour mieux appréhender les enjeux de leur développement. Le sociologue y contribue en élaborant une grille de lecture de la réalité sociale qu'il s'agit ensuite de modéliser. Attentif au projet social originel il questionne l'adéquation entre la modélisation d'accompagnement et ses usages sociaux. Les théories sociologiques sont ici confrontées aux réalités du terrain. Ce pragmatisme revisite la posture sociologique. 


\title{
Positionnement des sociologues dans la démarche de modélisation Domino'
}

\author{
WILLIAM'S DARÉ \\ Centre de coopération internationale de recherche \\ agronomique pour le développement (CIRAD), Paris, France \\ Christine Fourage \\ Université catholique de l'Ouest-CERIPSA \\ Angers, France \\ IBRAHIMA DIOP GAYE \\ École nationale d'économie appliquée (ENEA) \\ Dakar-Fann, Sénégal
}

1. Projet Domino et les enjeux d'une recherche « impliquée »

"Ah qu'il est loin le temps de la planification territoriale où les choix d'aménagement étaient simples, centralisés et rapides!»

Les principes de la gestion décentralisée des ressources adoptés par de nombreux pays leur imposent de prendre en compte la variété des

1 Le présent article n'aurait pas pu voir le jour sans l'appui réflexif des membres de l'ATP Domino du CIRAD appartenant à différentes institutions dont, notamment, l'ENEA de Dakar et l'UCO d'Angers. Domino réunit les trois auteurs de cet article et leur fournit, pour une part, le cadre de leur exercice scientifique. Par ailleurs, nous sommes redevables au collectif ComMod auquel, pour certains, nous participons. Il nous permet de confronter nos pratiques et de maintenir un dialogue méthodologique et théorique fructueux. 
parties prenantes, chacune pouvant avoir son point de vue sur les ressources en fonction de ses usages et de ses enjeux propres mais également de son échelle d'intervention ou de " concernement ${ }^{2}$ ». Parfois complémentaires, ces enjeux sont bien souvent contraires. Comment dans un tel contexte de complexité les gestionnaires de ces systèmes arrivent-ils à prendre « la bonne » décision?

De nombreux outils de représentation des dynamiques d'occupation des terres incluant partiellement leur impact sur les ressources naturelles ont été réalisés ${ }^{3}$, mais peu sont intelligibles par les acteurs eux-mêmes. Aider ces acteurs à gérer durablement leur environnement nécessite d'appréhender les interactions entre niveaux d'organisation aussi bien écologiques que sociaux ${ }^{4}$.

Le projet Domino (Démarche Objet Multisite pour l'étude des Interactions entre Niveaux d'Organisation) se focalise sur les processus d'affectation des terres au Sénégal et à La Réunion. L'enjeu est important. Il s'agit 1) de montrer que la construction d'un modèle d'affectation des terres peut intégrer les interactions entre acteurs/institutions dont les échelles de " concernement " sont héterogènes et 2) de questionner la généricité des modèles d'affectation des terres dans deux situations différentes : celle d'un département d'outre-mer français et celle d'un pays africain en développement.

Le sociologue dans cette démarche porte son regard sur les enjeux sociaux de la modélisation, sur le changement social qu'elle souhaite induire chez les participants du processus (voire chez les acteurs extérieurs représentés ou non). Mais il est également acteur du processus de modélisation. Quel est alors son rôle dans la dynamique qu'il

2 Luc Boltanski et Laurent Thévenot, De la justification : les économies de la grandeur, Paris, Gallimard, 1991.

3 Voir les modèles CLUE à http ://www.dow.wau.nl/clue ; Klaus Bellmann, «Towards to a system analytical and modelling approach for integration of ecological, hydrological, economical and social components of disturbed regions ", Landscape and urban planning, vol. 51, 2000, p. 75-87 ; ou Dawn Parker et al., « Multi-agent systems for the simulation of land-use and land-cover change: A review ", Annals of the Association of American geographers, vol. 93, $\mathrm{n}^{\circ} 2,2003$, p. 314-337.

4 Crawford S. Holling, «Understanding the complexity of economic, ecological and social systems », Ecosystems, n" 4, 2001, p. 390-405. 


\section{WILLIAM'S DARÉ... / POSITIONNEMENT DES SOCIOLOGUES...}

souhaite analyser? Qu'attendent les autres protagonistes de la présence d'un sociologue parmi eux ? Quelles sont les limites à son exercice?

\section{Des contextes différents, un même souci de la sécurisation du foncier}

\subsection{Usages multiples de l'espace}

À La Réunion, la maittrise du foncier est une question cruciale. L'espace de La Réunion est limité. Sur les 252000 ha que comptent lîle, $20 \%$ sont occupés par de l'agriculture, $34 \%$ par des espaces naturels boisés, $10 \%$ par des logements, $21 \%$ par des espaces non exploités (type landes ou friches) et le reste est non exploitable 5 . Or, avec une croissance démographique prévue de $33 \%$ sur vingt ans (950 000 habitants en 2020 contre 710000 en 1999), c'est près de 7000 ha qui devraient être mobilisés pour répondre à la demande de logements. Les réserves foncières constituent seulement $1100 \mathrm{ha}^{6}$. La projection des besoins supplémentaires en foncier des différentes filières agricoles est estimée à 6650 ha, ces extensions pouvant être majoritairement réalisées sur des terres actuellement en friches ${ }^{7}$. Dans cette dynamique, malgré des objectifs de développement ou plus simplement de maintien des filières agricoles, le foncier qui était dévolu à l'agriculture et aux espaces naturels est progressivement grignoté par le développement anarchique de l'urbanisation.

Au Sénégal, les cinq communautés rurales autour du lac de Guiers composent la zone d'étude. Le développement rapide de l'agriculture irriguée exerce une pression accrue rendant difficile l'accès du bétail aux pâturages. Les rejets industriels et agricoles détériorent la qualité de l'eau dégradant les ressources halieutiques disponibles pour les pêcheurs. La fourniture en eau potable pour les populations des villes recherchée par

5 Agreste. Mémento agricole : la statistique agricole, Ministère de l'agriculture et de la pêche, Direction de l'agriculture et de la forêt de La Réunion, Michel Sinoir (dir.), SaintDenis, La Réunion, 2005.

6 Schéma Départemental d'Aménagement et de Développement Durable (SDADD), Le diagnostic stratégique et les approches territoriales, Saint-Denis, Département de La Réunion, septembre 2005.

7 Ibid. 
l'État se traduit par une concurrence sur les quantités. Il existe une grande variété des modes d'accès à la terre (droit moderne, don, prêt, héritage, achat et location) hypothéquant une sécurisation du foncier. De nombreux cas de litiges et de conflits fonciers sont notés au travers des multi-affectations d'une même parcelle, des affectations à des personnes non identifiées, des difficultés à désaffecter, et de l'inexistence de cadastre rural.

\subsection{Des acteurs nombreux ayant des usages de l'espace complémen-} taires ou antagonistes

À La Réunion, les dispositifs réglementaires de planification de l'aménagement du territoire aux différentes échelles de gestion depuis la commune (avec son Plan Local d'Urbanisme), jusqu'à la région (avec son Schéma d'Aménagement Régional [SAR]) en passant par les Communautés de Commune (et leurs Schémas de Cohérence Territoriale [SCOT]) sont en phase soit de révision, soit d'élaboration. Issus de la Loi sur la solidarité et le renouvellement urbain de 2000, ces dispositifs doivent appliquer les principes d'une démocratie participative, recueillir et intégrer lors de processus de concertation les points de vue, enjeux et contraintes des multiples acteurs concernés (membres de la société civile, des institu-tions ayant mandat sur la gestion du territoire ou de ses ressources, etc.). Mais en fonction des mandats des décideurs politiques et des pressions qu'ils subissent, chacun définit ses objectifs prioritaires - entre préservation du milieu naturel, développement urbain, maintien des filières agricoles, ou création d'emplois -, partageant ensuite son espace limité entre les activités correspondantes (élevage, canne à sucre, tourisme dans les espaces naturels et sur la frange littoral, etc.).

Au Sénégal la présence du lac attire des acteurs divers et de plus en plus nombreux. Car la terre n'a en soi pas de valeur sans droits d'accès à l'eau. Le Conseil rural, organe délibérant de la communauté rurale depuis 1972, est le seul habilité à gérer les terres des « zones de terroir». Avec la loi n ${ }^{\circ} 96.07$ du 22 mars 1996, il les affecte aux membres de la Communauté rurale qui les mettent en valeur sous le contrôle de l'État, représenté par le sous-préfet. Le Conseil rural s'est vu transférer neuf autres domaines de compétences parmi lesquels la gestion des ressources naturelles et l'environnement, l'éducation, la santé, l'urbanisme 
et l'aménagement du territoire. Il reste toutefois limité dans son action par la faiblesse de ses moyens financiers et techniques. Dès lors, la tendance à la disparition de la jachère, la dégradation des terres et la rude compétition foncière suite à l'arrivée progressive de grands exploitants nationaux et étrangers, seuls en mesure de supporter le coût prohibitif de la mise en valeur dans la zone, compliquent davantage l'accès au foncier des petits exploitants familiaux et plus particulièrement des femmes ${ }^{8}$.

\subsection{Enjeu de la sécurisation du foncier}

Comment mettre en œuvre une politique foncière d'aménagement du territoire durable qui prenne en compte le caractère limité de l'espace et les besoins croissants des différentes activités consommatrices de foncier? Telle est la question centrale posée dans les débats actuels sur l'aménagement du foncier réunionnais. Dans le contexte sénégalais, de plus en plus inégalitaire, la survie des communautés villageoises est fortement conditionnée par la réponse à la question suivante : comment garantir la coexistence durable des systèmes d'exploitation et la viabilité de la petite exploitation dans la zone du lac de Guiers?

\subsection{Attendus du projet Domino : modélisation participative des} interactions à l'œuvre dans la gestion du foncier

Le projet Domino vise à représenter les interactions entre les dynamiques foncières urbaines, agricoles et naturelles. Mais cette représentation nécessite de considérer les espaces de concernement des acteurs et institutions - région/département, intercommunalité, commune, voire infra-communal - qui participent à l'aménagement du territoire et leurs interactions. Il s'agit également de promouvoir une démarche de modélisation participative pour penser la gestion du

8 Un pour cent des affectations sont faites à des groupements de femmes et aucun cas d'affectation individuelle n'a été recensé (selon les déclarations des présidents des conseils ruraux de Syer, Keur Momar Sarr et Mbane - trois communautés rurales autour du lac - à l'atelier de mise en place du comité de suivi Domino). 
territoire visant à accroître la résilience ${ }^{9}$ des anthroposystèmes associés ${ }^{10}$. Cette démarche s'appuie sur trois hypothèses :

- l'intégration des parties prenantes dès les premières étapes d'un processus de modélisation est nécessaire à la légitimation de l'outil produit et à son appropriation par les acteurs ;

- le modèle est un outil médiateur autour et à propos duquel s'échangent les points de vue des différents participants de la modélisation ;

- l'explicitation des hypothèses de construction du modèle et la mise en interaction des éléments du système de gestion dans un modèle informatique de type multi-agents permettent de construire et tester des scénarii prospectifs de gestion.

Les sociologues dans Domino se concentrent sur la construction sociale du modèle par une communauté qui réunit des chercheurs de différentes disciplines, des acteurs producteurs d'information et des utilisateurs (gestionnaires et décideurs). Chacun participe au processus de modélisation en y apportant son propre point de vue sur le système socio-écologique étudié. Cette démarche est héritée de la modélisation d'accompagnement développée par le groupe ComMod et d'autres chercheurs $^{11}$. C'est sur cette pratique de modélisation, qui cherche à

9 Nous nous référons ici au sens écologique de résilience, c'est-à-dire à la capacité d'un système à absorber des perturbations tout en conservant sa structure et en assumant sa fonction. Cette notion s'applique à des systèmes complexes pouvant avoir plusieurs états ou domaines d'équilibre. Enfin, elle se caractérise par le degré d'autoorganisation du système et par ses capacités d'adaptation et d'apprentissage. Voir : Crawford S. Holling, "Resilience and stability of ecological systems », Annual review of ecology and systematics, $\mathrm{n}^{\circ}$ 4, 1973, p. 1-23 ; Crawford S. Holling, "The resilience of terrestrial ecosystems; local surprise and global change, dans William C. Clark et R. E. Munn (dir.), Sustainable development of the biosphere, Cambridge (U.K.), Cambridge University Press, 1986, p. 292-317 ; Crawford S. Holling, "Engineering resilience versus ecological resilience ", dans Peter Schulze (dir.), Engineering within ecological constraints, Washington (D.C.), National academy, 1996, p. 31-44.

10 On entend par anthroposystème l'ensemble des écosystèmes et sociosystèmes en interactions sur un espace donné, le fonctionnement de l'anthroposystème comprend donc les dynamiques sociales et environnementales ayant lieu sur cet espace.

11 Collectif ComMod, "La modélisation comme outil d'accompagnement ", Nature, sciences et sociétés, vol. 13, n" 2, 2005, p. 165-169 ; Brian Walker et al., " Resilience management in social-ecosystems: A working hypothesis for a participatory approach », Conservation ecology, vol. 6, n" 1, 2002, 14 p. ; Robert M. Argent et Rodger 
expliciter les hypothèses et à favoriser les échanges d'informations entre les différents protagonistes, que portera notre regard. La modélisation d'accompagnement est une posture scientifique qui caractérise une recherche «impliquée » dans le développement. La démarche consiste en des allers-retours entre les théories et les terrains d'étude. Le chercheur adoptant cette posture scientifique post-normale ${ }^{12}$ accompagne les parties prenantes lors de la négociation ${ }^{13}$. Il s'intéresse au processus de décision qui aboutira, pas à pas, à l'établissement d'une solution acceptée, légitimée et viable.

\section{Sociologues : observateurs ou participants?}

Dans Domino, des sociologues sont impliqués sur chacun des sites. Ils interviennent à plusieurs titres. Tout comme les autres «thématiciens » ils apportent aux groupes leurs connaissances des dynamiques sociales de gestion des territoires.

À La Réunion, ils ont participé à l'identification des acteurs clés dans divers processus de concertation autour de la gestion des ressources. Par exemple, en utilisant les différentes techniques classiques de recueil de données (enquêtes et observation participante, notamment), ils ont pu décrire les interactions entre acteurs impliqués dans la gestion des espaces agricoles aux échelles communale ou départementale (le niveau intercommunal est prévu pour 2006-2007). À l'échelle communale, la question du devenir du foncier agricole à l'horizon 2020 est explicitement posée dans le cadre des chartes de développement agricole initiées par la Chambre d'Agriculture de La Réunion. Ce travail vise à rassembler l'ensemble des acteurs du secteur agricole sur une commune et à définir les enjeux actuels de l'agriculture dans leur zone, leurs objectifs dans quinze ans, et leur traduction sous la forme de fichesaction à mener pour atteindre les buts exprimés. Cette démarche, bien

B. Grayson, « Modelling shell for participatory assessment and management of natural resources », Environmental modelling and sofiware, vol. 18, n 6, 2003, p. 541-551.

12 Silvio O. Funtowicz et Jerry R. Ravetz, « Science for the post-normal age », Futures, vol. $25, \mathrm{n}^{\circ} 7,1993$, p. $739-755$.

13 Cette position constructiviste de la négociation permet d'en suivte pas à pas le déroulement. Voir Jean-Louis Le Moigne, Le constructivisme : les fondements, vol. 1, Paris, ESF, 1994. 
que contractualisée entre les parties, n'est pas réglementaire, et seul le contrôle social local peut permettre de la faire respecter. Ces analyses viennent en complément des nombreux travaux réalisés à La Réunion. D'autres études sont menées afin de mieux comprendre le contexte sociopolitique dans lequel sont prises les décisions d'aménagement du territoire.

Au Sénégal, dans la région du lac de Guiers, une première analyse sociopolitique a permis de montrer que non seulement les acteurs sont multiples mais qu'en plus certains sont hybrides. Hybrides parce qu'ils cultivent la multi-appartenance institutionnelle : chaque institution possède son point de vue sur le foncier en faisant référence à des corpus de normes propres au système coutumier ou au système moderne. Dans ce contexte, la décentralisation se présente comme le fruit d'un compromis entre référentiel de maintien de la tradition et référentiel de modernisation. Ce dernier se caractérise par la production de normes (administratives, planificatrices, etc.) et par le contrôle de la légalité, ce qui ouvre une quantité d'espaces de négociation entre représentants de l'État local et élus locaux, notamment dans les critères de mise en application (décrets, arrêtés, etc.) des différentes productions législatives (Loi sur le domaine national, Loi sur l'orientation agrosylvopastorale, Code de l'eau, entre autres).

La modélisation permet d'intégrer les différents aspects thématiques de l'affectation des terres tout en poussant à la schématisation de la complexité représentée. Cette schématisation impose à chaque protagoniste de définir clairement ses enjeux mais aussi d'identifier les éléments fondamentaux pour leur intégration dans le modèle.

Initialement appelé pour contribuer à l'élaboration d'une grille de lecture des dynamiques sociales en relation avec les dynamiques écologiques qui seront ensuite modélisées, le sociologue ne peut s'arrêter là. L'analyse qu'il réalise ne peut être cantonnée à l'extérieur du processus de modélisation comme celles des autres thématiciens. Il devra être attentif à ce que la construction du projet ne soit pas basée seulement sur les hypothèses du travail collectif où chaque partenaire instille un peu de ses attentes, mais qu'elle soit le fruit d'hypothèses scientifiques, expliquées pas à pas et enrichies par tous les participants. C'est la rigueur scientifique du sociologue qui se trouve mobilisée. Il est de sa responsabilité d'ouvrir à des perspectives plus larges que les perspectives parcellaires des différents acteurs pris séparément, afin de 


\section{WILLIAM'S DARÉ... / POSITIONNEMENT DES SOCIOLOGUES...}

tresser une maïeutique de recherche collective, qui soit aussi une maïeutique sociale ${ }^{14}$. Il s'agit de contribuer à produire collectivement une orientation problématique qui n'est pas la somme de toutes les approches mais bien le produit maitrisé d'une conception globale de la question à traiter.

Par sa connaissance des parties prenantes et de leurs enjeux, il éclaire leurs prises de position, décrypte leurs stratégies d'intervention, révèle l'origine des tensions ou des alliances apparues au cours du processus de modélisation. Or, l'un des enjeux de cette recherche qui utilise comme support la co-construction de l'outil de modélisation est bien d'accroître la connaissance du système de gestion de chaque acteur. Cela signifie les aider à mieux saisir les enjeux techniques de l'affectation des terres et faciliter les échanges de points de vue pour une plus grande compréhension des enjeux sociaux qui animent chacune des parties prenantes.

Le chercheur en sociologie, membre actif d'un collectif d'accompagnement, doit être attentif à la volonté réformatrice du projet social à l'origine de l'outil. Son implication dans Domino vise également à questionner l'adéquation entre la modélisation et ses usages sociaux avec, comme enjeu, le changement dans les routines opérationnelles. Cette question est centrale, elle est rarement envisagée pour elle-même parce que la dynamique collective rend difficile l'interrogation sur le produit qui se dessine. Les partenaires du projet sont embarqués dans une aventure humaine en même temps que scientifique, où l'innovation exalte les esprits. La légitimité du projet passe par l'action et l'adhésion, lesquelles renforcent le lien. Ne pas ouvrer dans le sens du collectif, c'est en quelque sorte le trahir. Les critiques sont indicibles et impensables lorsque qu'elles se penchent sur le coût méthodologique de l'approche, sur la réalité de l'appropriation par les acteurs non scientifiques. Il y a un effort non négligeable à entreprendre pour la transmission des savoirs scientifiques. Effort de longue haleine dont on n'est pas toujours certain qu'il suscite l'adhésion d'acteurs sociaux (notamment de décideurs) pris bien souvent dans des échéances à court terme. Engagés dans des enjeux institutionnels requérant une opérationnalité

14 Michel Verret, Chevilles ouvrières, Paris, Les éditions de l'atelier et Les éditions ouvrières, 1995. 
immédiate, leur pas de temps n'est pas celui de la recherche. Quant aux acteurs bénéficiaires du projet, il est vraisemblable que leurs attentes ne s'expriment pas dans les mêmes termes que celles des chercheurs. Ce sont pourtant celles des «populations-cibles » qui devraient être au cœur des interrogations de la recherche impliquée.

En droite ligne des grands principes de la participation, cette démarche vise à augmenter la « capacitation ${ }^{15}$ » des parties prenantes. Mais derrière ce vocable se cache de nombreuses dérives, telle celle de la «fausse participation ${ }^{16}$ ", c'est-à-dire une participation où toutes les parties ne prendraient pas part à la construction. L'identification de toutes les parties prenantes dès le début de la modélisation est une étape essentielle mais non suffisante car certaines peuvent très bien avoir été oubliées. La démarche de modélisation participative par les allers-retours qu'elle propose et son principe d'échange d'informations devrait permettre d'éviter cet écueil. Il est indispensable que les protagonistes ne considèrent pas la démarche comme imposée, comme un processus déjà ficelé auquel ils ne feraient que «prendre part». C'est pourquoi la modélisation doit être considérée comme légitime par l'ensemble des acteurs pour leur permettre d'accepter la pertinence des autres points de vue sur les modalités de prise de décision concernant l'affectation des terres. Sans cette légitimité établie dans la confiance de la co-construction du modèle " terrain », on ne peut pas favoriser les interactions et la coordination des différents niveaux d'organisation. L'analyse des changements sociaux induits par la participation des acteurs au processus de modélisation est un point essentiel de l'intervention du sociologue dans Domino ${ }^{17}$.

15 Traduction française de la notion d'empowerment qui sous-entend l'acquisition et l'utilisation d'outils et de connaissances, la prise de conscience de sa valeur et la capacité de changer les choses, le tout en passant par l'action concrète et conduisant ultimement à l'exercice d'une influence significative sur son environnement et sa condition.

16 Philippe Lavigne Delville, Nour-Eddine Sellamna et Marilou Mathieu (dir.), Les enquêtes participatives en débat: ambition, pratiques et enjeux, Paris, Karthala / ICRA/ GRET, 2000.

17 Mais ce travail d'évaluation sociologique de l'influence de Domino sur les modalités de prise de décision publique sur l'affectation des terres est un axe de recherche en soi. D'autres équipes de recherche réalisant également des démarches de modélisation 
La question de la neutralité du sociologue engagé dans Domino, n'a plus lieu d'être. Il est un observateur impliqué. Il participe au processus de dévoilement, à la circulation de l'information entre les différents protagonistes et, ce faisant, il contribue à la modification des rapports sociaux entre les individus et à propos de leur environnement.

$\grave{A}$ travers sa participation, le sociologue en intervention participe à l'élaboration d'un « microcosme commun que cisèlent ses interlocuteurs et auquel lui-même se trouve attaché ${ }^{18} \gg$. Il s'agit de constituer un espace d'intéressement en tant que lieu de circulation de savoir, circulation portée par des acteurs issus de mondes différents et qui impulsent la coconstruction de nouveaux savoirs ${ }^{19}$. C'est certainement ce qui peut paraitre le plus ardu dans la modélisation d'accompagnement, qui requiert l'assentiment de tous à tout moment et qui, de ce fait, alourdit la progression commune quand elle ne l'entrave pas momentanément, semblant conduire à des impasses par défaut d'adhésion. À La Réunion, certains acteurs de terrain sont jugés centraux dans la description des processus et dynamiques que l'on souhaite modéliser. Mais la modélisation impose une schématisation donc une simplification difficilement compatible avec la multiplicité des situations singulières à laquelle ils sont quotidiennement confrontés. Le passage à l'abstraction exigé par le travail de conceptualisation des modèles n'est pas une évidence et nécessite des ajustements progressifs des différentes parties. Pourtant, ce travail de fourmis, de négociation/explicitation pas à pas, est essentiel pour l'adhésion des individus au groupe des "modélisateurs ». Le modèle devenant celui de ce collectif, c'est en tant que membres qu'ils justifieront ensuite les options prises face à des tiers lors des discussions autour de scénarii prospectifs d'aménagement du territoire.

d'accompagnement pour la gestion des ressources naturelles se sont associées pour se saisir de cette question et construire un projet de recherche commun pour y répondre (ComMod). Domino est l'un des terrains d'étude et les résultats de cette recherche sont attendus pour 2008.

18 Gilles Herreros, Pour une sociologie d'intentention, Paris, Érès, 2002.

19 Madeleine Akrich et al., « À quoi tient le succès des innovations ? 1 ; L'art de l'intéressement 2 : Le choix des porte-parole ", Gérer et comprendre. Annales des mines, $\mathrm{n}^{\prime \prime} 11,1988$, p. $4-17$ et $\mathrm{n}^{\prime \prime} 12,1988$, p. 14-29. 


\section{Intérêts de l'intervention de sociologues dans le projet}

La modélisation devrait fournir aux acteurs, des outils, des techniques ou de l'information sur le champ des possibles de leur environnement. Le sociologue, observateur et participant, doit s'engager à accompagner les acteurs dans la perception du devenir de leur territoire parce que les espaces ici considérés sont symboliquement investis, économiquement gérés et politiquement encadrés.

La question de la pertinence de l'intervention du sociologue dans ce type de projet se pose. La démarche de modélisation puis d'implémentation informatique d'un modèle commun de simulation de la réalité sociale atteint, il faut en convenir, un degré de réification sans commune mesure avec les dispositifs méthodologiques classiques. Le péril est grand de "prendre les artefacts pour les faits »!

\subsection{Défis de la pluridisciplinarité}

Le chercheur en sciences sociales intervient, en premier lieu, en amont de la modélisation (même s'il participe activement à son élaboration et à sa mise en ouvre). On lui demande de fournir les pré-requis, de décrire le contexte social, les interactions entre acteurs, de révéler les champs de force et de faiblesse, les mécanismes de prise de décisions, etc. Il sait qu'il doit sensibiliser ses partenaires à la complexité sociale, qui, bien sûr, est multiforme. Il tentera de les convaincre de la nécessité de se pencher sur de multiples aspects (culturels, religieux, symboliques, politiques, socio-dynamiques, etc.) que les participants peuvent considérer comme secondaires privilégiant les dimensions de la gestion et de la planification. Par ses interactions avec les autres acteurs, il contribue à inscrire un savoir ou plutôt une culture technique dans une sociabilité plus large. Car les hommes et les femmes impliqués dans Domino ne se limitent pas à quelques professionnels privilégiés, ils constituent une gamme assez vaste de personnes d'appartenances et d'horizons très divers, qui partagent l'aventure de la modélisation. À La Réunion comme au Sénégal, se côtoient au sein du groupe de modélisation des chercheurs de disciplines et d'institutions différentes, françaises et sénégalaises (instituts de recherche agronomique, universités, etc.), des 
agents des administrations publiques ou assimilées et des membres de la société civile directement intéressés par l'aménagement du territoire ${ }^{20}$.

Cependant, malgré cette volonté d'impliquer les acteurs dès le début du processus de modélisation, il faut être réaliste et reconnaître les limites objectives de chacun. Dans Domino, même si le modèle a largement été débattu et élaboré par les acteurs scientifiques et certains acteurs institutionnels (chambre d'agriculture, communauté rurale, acteurs « relais », etc.), le passage à la formalisation est dévolu aux seuls modélisateurs, censés être l'interface entre les experts scientifiques et les acteurs de terrain. L'implémentation reste du ressort des informaticiens. On invoque des contraintes de temps, des contraintes institutionnelles ou financières (certes réelles) pour exclure de fait certains partenaires non scientifiques. Dès lors, on peut s'interroger sur les limites de la coconstruction.

La participation des sociologues aux groupes de travail de modélisation a pour ambition de permettre à l'ensemble des acteurs de dépasser le cadre des évidences et d'enrichir les modèles. Il s'agit bien de décrire au mieux la réalité sociale, mais, ici comme ailleurs, on ne fait que l'approcher. Le rôle des sciences sociales est de faire que cette approximation soit la plus pertinente possible, qu'elle rende justice aux conditions sociales faites aux acteurs au profit de qui le projet est initié et qui doit les servir.

Du point de vue de la sociologie classique (comme d'ailleurs du point de vue de toutes les autres disciplines), l'échange pluridisciplinaire et pluri-acteurs ne peut s'incarner que dans le consensus a minima, autour d'une vulgate peu propice à l'ambition théorique. De fait, la coconstruction du modèle d'analyse suppose une procédure itérative d'où, peu à peu, un savoir commun aux différents participants émerge, mais où chacun sait qu'il abandonne une portion de sa vérité disciplinaire et de son sens commun. Il faut faire le deuil de l'hégémonie des sciences sociales pour se fondre dans une représentation partagée d'une question sociale à traiter et à résoudre. La complexité technique de l'approche, si elle est stimulante sur le plan intellectuel, comporte le risque, pas toujours assumé, d'un basculement vers une sorte d'ingénierie sociale ou

20. En raison de leur faible disponibilité, les décideurs politiques ne seront impliqués que dans la phase prochaine de proposition/test de scénarii d'affectation des terres. 


\section{NPSS, VOLUME2, NUMERO 2, 2007}

informatique susceptible de prendre le pas sur la conquête et la maîtrise de l'objet.

\subsection{L'analyse sociologique soumise à l'épreuve de la modélisation}

L'analyse sociologique des phénomènes sociaux peut se trouver perturbée par une instrumentalisation et une injonction (demande) forte de validation des processus à l'œuvre. Se transformant en expert de la chose sociale, le sociologue apporte sa caution au projet scientifique et à son utilisation par les décideurs (institutionnels et politiques) engagés dans une dialectique de l'intérêt général mais réticents face aux questionnements ayant trait à leur domination " naturelle et non consciente ». L'appropriation et la participation à cette expérience ne sont pas neutres ; la désignation d'acteurs privilégiés, de personnes ressources se doit d'être interrogée. Le sociologue peut également aider à davantage saisir l'antagonisme des logiques qui s'expriment ou qui restent implicites mais agissantes. Dans le territoire du lac de Guiers, se superposent à la logique planificatrice des technocrates, celle de négociation - participation des organisations de producteurs -, celle du marché foncier (bien que non légalisée) et celle dite coutumière.

Certes, l'apport de la sociologie peut se trouver réduit dans le processus de modélisation. Cette réduction est-elle insurmontable ? Pourquoi participer à une expérience où l'on vend son âme au diable (comme a tendance à le penser la sociologie française des années 1970)? Parce que nous sommes des « fabricants du social ». Cela semble évident dans une recherche appliquée et impliquée mais cela est toujours le cas parce qu'en décrivant les objets nous les transformons, nous les affectons $^{21}$ et nous ne contrôlons pas l'effet de nos productions. L'analyse sociologique serait bien naive $\mathrm{e}^{22}$ si elle occultait que la posture d'accompagnement induit des changements sociaux.

21 Anthony Giddens, The constitution of society, Cambridge, Polity Press, 1986.

22 Jean-François Sirinelli, en collaboration avec Pascal Ory, Les intellectuels en France, de l'affaire Dreyfus à nos jours, Paris, Armand Colin, 2002. 


\section{3. Éthique du lien, réalisme utopique et engagement}

Nous suggérons une perspective pragmatique marquée par «le recours à une éthique du lien et à un réalisme utopique $e^{23} \gg$. La réussite de la modélisation d'accompagnement passe par une mise à plat des hypothèses scientifiques des uns et des autres, la conviction partagée (et sans cesse à affirmer et à retravailler) du bien-fondé de tous les éclairages des participants (experts et acteurs) à la démarche, sans qu'aucun n'ait $a$ priori la prééminence. L'enjeu est la coopération, librement consentie, des bénéficiaires du projet scientifique à ses attendus, et leur appropriation afin de « sécuriser » leur devenir au moindre coût social. Il s'agit de mettre en place des espaces d'élaboration, de discussion et de négociation du projet réduisant, autant que faire se peut, les effets d'imposition et de violence symbolique. Cette coopération, cette volonté tendue vers un même objectif - la gestion et la maitrise d'un environnement viable pour le plus grand nombre - repose sur un façonnage d'un lien social dense, horizontal permettant la communication et l'échange en minimisant les effets de la suprématie des savoirs et des discours sur un objet (ou autour d'un objet). C'est ce que nous désignons par éthique du lien social. Il faut prendre appui sur les synergies existantes, les valoriser et les mettre à profit dans le projet de recherche.

Le réalisme utopique, quant à lui, consiste à clairement contrôler et défendre les apports méthodologiques de la sociologie en insistant sur les savoirs-faire dont la discipline s'est dotée au fil du temps en tant que discipline empirique, et en les mettant à la disposition des acteurs de la démarche partageant des objectifs de recherche et d'utilisation des produits de celle-ci. Le sociologue, pour y participer pleinement, doit se saisir des outils de la modélisation et comprendre a minima les perspectives des autres disciplines ${ }^{24}$. Dans le même temps, il se doit d'être ferme sur ce qui lui semble essentiel à transmettre de son

23 Gilles Herreros, Pour une sociologie d'intervention, Paris, Érès, 2002.

24 Il est important que le sociologue soit en mesure de pénétrer les tenants et les aboutissants de la modélisation sans se substituer aux modélisateurs. Il devra néanmoins pouvoir saisir les arcanes de la formalisation afin de veiller à ce que la phase de représentation du modèle prenne en compte du mieux possible les hypothèses théoriques propres à sa discipline. 
expérience scientifique en réalisant un effort pédagogique qui le conduit à abandonner un jargon conceptuel et problématique si celui-ci ne peut pas, en l'état, être approprié par les partenaires du projet. Il participe à la mise en place d'une communauté de vues, d'échanges, de liens où la coopération est le maître mot. Il ne s'agit pas d'endosser l'habit du " militant», sa fonction doit avant tout demeurer celle de façonner, dans le cadre d'une coopération, dans des situations particulières, des connaissances mobilisables par le plus grand nombre.

Il faut dépasser le débat entre engagement et distanciation, pour revendiquer un engagement scientifique humaniste et pragmatique. Ce sera l'occasion d'accorder une plus grande importance aux pratiques de la démonstration et de participer à faire de la modélisation un véritable langage social. Le projet Domino s'intègre dans la perspective du développement durable (notion certes polysémique et discutable), qui est ontologiquement un parti pris idéologique et une vision du monde. L'engagement n'est pas une trahison et nous permet d'assumer un héritage historique, celui des fondateurs de la sociologie française et américaine (entre autres) ; c'est une posture dont nous nous revendiquons.

Une occasion unique est donnée au sociologue de tester en temps réel et en "grandeur nature " les théories qu'il a contribué à élaborer. L'ajustement théorique aux réalités du terrain devient l'un des enjeux de sa participation au projet. Le pari est davantage social que scientifique et intellectuel. Il peut résider dans la contribution à l'engagement collectif au détriment de l'innovation dans le domaine des sciences sociales. Le sociologue ne sait pas mieux que ceux parmi lesquels il intervient ce qui est « juste » ou «bon » pour eux mais, appuyé sur ses options méthodologiques, acceptant son engagement dans la situation, il apporte sa pierre à la fabrication d'un matériau susceptible de constituer une aide pour ses interlocuteurs. Sa crédibilité est à ce prix, crédibilité en tant que chercheur, parce qu'il peut être démenti à tout moment par la réalité qu'il tente de décrire, crédibilité en tant qu'acteur du projet par ce qu'il est, au même titre que les autres membres de l'équipe, engagé dans sa réussite. 


\section{Pragmatisme qui n'est pas sans poser de difficultés}

Le sociologue impliqué se trouve confronté à la réalité des autres partenaires de la modélisation. Ce pragmatisme interroge sa posture, son rapport aux membres du projet et aux populations concernées par la démarche d'accompagnement.

\subsection{Aux sociologues}

Les repères habituels du chercheur qui recueille ses données dans la solitude sont brouillés. Le processus sociologique classique suppose que l'acteur social ne " connaît pas le sens de ses actes ${ }^{25}$ ». Or la démarche de modélisation d'accompagnement requiert une explicitation de tous les instants des pratiques sociales remettant en cause l'extériorité par rapport aux choses (perspective durkheimienne) du chercheur en sciences sociales. Cette même exigence est présente dans le processus d'analyse des informations de terrain, risquant de parasiter l'analyse sociologique afin de dégager un consensus pluridisciplinaire et pluriacteurs. Il faut se forger autre chose qu'une éthique de l'interdisciplinarité et recourir à une réelle modestie quant à l'apport pluridisciplinaire «de façade » confronté à des types de savoirs pluriels tout aussi légitimes les uns que les autres.

Le sociologue en tant que participant au processus de modélisation devient détenteur d'un savoir parmi d'autres au même titre que les scientifiques ou les experts "profanes ». Dès lors, sa position d'intériorité (en tant que participant du processus de modélisation) et d'extériorité (en tant qu'observateur analysant le processus en cours) le place dans une situation plus qu'ambiguë vis-à-vis des autres membres du groupe de modélisation mais également de son groupe d'appartenance disciplinaire. Ne risque-t-il pas d'être juge et partie ? Pour satisfaire aux exigences de sa discipline, le sociologue doit répondre à une double exigence : un impératif de réflexivité sur sa propre implication dans la démarche tout en favorisant la prise de conscience

25. Pierre Bourdieu, Questions de sociologie, Paris, Minuit, 1980 ; Pierre Bourdieu et Loï Wacquant, Réponses : pour une anthropologie réflexive, Paris, Seuil, 1992. 
par les autres acteurs de la pluralité des mondes réduits dans le modèle $e^{26}$. Cependant, la mise en commun des savoirs scientifiques à mobiliser pour décrire un système complexe ne risque-t-elle pas de conduire à une sorte de réification scientifique de la réalité sociale ? L'explicitation des hypothèses de la modélisation et leur mise en débat au sein d'un groupe restreint d'utilisateurs devrait permettre de limiter cet écueil.

\subsection{Aux autres participants (acteurs, chercheurs : «thématiciens » et modélisateurs)}

La difficulté la plus importante réside dans la prise en charge de pas de temps, d'échelles, de points de vue différents qu'il faut intégrer, concerter, négocier, exclure au cours du processus de modélisation. L'analyse sociologique servira aux "thématiciens " pour faire les choix les plus pertinents possibles du point de vue de l'objectif final de l'exercice de modélisation, à savoir un accompagnement des acteurs vers une gestion plus durable de leur espace, ce qui ne saurait éluder la question des rapports de force à côté d'un éventail de droits de différente nature qui marquent l'accès et l'usage de la terre.

Au Sénégal, la gestion du foncier repose sur une abondance de textes législatifs qui définissent différents secteurs et qui sont élaborés de manière sectorielle : Code de l'eau, Code de l'hygiène, Code forestier, Code de l'environnement, Code du domaine de l'État, Décret portant sur l'organisation des parcours et fixant les conditions d'utilisation des pâturages, Loi sur le domaine national, Code minier, etc., le dernier en date étant la loi agrosylvopastorale de juin 2004. Cette volonté d'organiser les rapports au foncier n'est pas sans susciter des problèmes d'application car ces instruments qui s'ignorent génèrent très souvent des conflits de prérogatives sur le terrain. Par exemple, la forêt classée de Richard Toll, forêt sans arbre, sans végétation ni faune, est située au cœur de la commune qui a besoin d'espace pour la construction de maisons. Le

26 Il est à noter cependant que nos travaux ont montré que, même si la modélisation induisait une réduction, la mise en débat du modèle avec des parties prenantes de l'anthroposystème représenté permet de restituer une partie de la complexité de la réalité sociale. Voir William's Daré, Comportements des acteurs dans le jeu et dans la réalité. Indépendance ou correspondance? Analyse sociologique de l'utilisation de jeux de róles en aide à la concertation, Thèse ENGREF, Paris, 2005. 
ministère des Finances (par la Direction des domaines) et le ministère de l'Urbanisme pour les opérations d'urbanisme interviennent sur cet espace dont le statut donne toujours prérogative à la Direction des eaux et forêts. Ici, deux ministères gèrent sans concertation des ressources localisées sur ce même espace.

À La Réunion, bien que le droit moderne soit le principal revendiqué, on assiste à une superposition de dispositifs réglementaires nationaux et européens qui régissent les usages de l'espace. Selon les différentes échelles du territoire, ces dispositifs peuvent avoir diverses vocations depuis l'intégration d'usages multiples sur un même territoire (schéma d'aménagement régional, schéma de cohérence territoriale) jusqu'à la protection d'un usage particulier (urbain, agricole ou espace naturel). L'interaction entre ces dispositifs paraît être réalisée par des acteurs mandatés pour réfléchir à leur mise en place. Peu nombreux comparativement à la métropole, acteurs hybrides, ils appartiennent en général à plusieurs groupes sociaux qu'ils représentent en fonction de la situation dans laquelle ils interviennent. Ils possèdent différentes casquettes qu'ils peuvent mobiliser et défendre au gré des sujets des réunions auxquelles ils sont conviés. Extrêmement sollicités, ils sont pris dans des interactions de court terme, ils n'ont que peu de temps à consacrer à une réflexion scientifique. Ils sont pourtant essentiels au projet Domino pour l'enrichissement thématique du projet et des participants et également en tant que leaders de groupe susceptibles d'initier des changements de société.

De ce point de vue, Domino est un montage de partenariats visant à développer des outils et à gérer des interfaces avec des résultats divers, que l'on espère prometteurs pour chaque participant. Il entend se fonder sur l'impartialité d'une entité (agrégat de plusieurs entités plus petites) qui observe plusieurs dynamiques (essentiellement foncières) en vue de mieux procéder à des articulations (par exemple entre législations et pratiques) négociées et acceptées. En tant que réflexion sur des contextes sociopolitique et écologique différents, le processus Domino apparaît comme une démarche visant à intégrer, à construire et à reconfigurer les compétences des participants en vue de leur permettre d'appréhender des environnements changeants auxquels ils doivent s'adapter et de contribuer à cette adaptation. 


\section{3. À la population cible}

L'entrée par des acteurs " relais » est la voie généralement privilégiée pour initier le processus de co-construction. Ces acteurs sont les représentants des groupes ou des institutions ciblés par Domino, chacun ayant des intérêts différents sur le foncier. Hybrides ou non, ces personnes ressources occupent des statuts sociaux « honorifiques ${ }^{27}$ ». Leur participation à la démarche peut renforcer leurs positions et servir leurs intérêts. Dès lors, l'implication des acteurs dans le processus de modélisation peut modifier localement les jeux de pouvoir, et être source de conflits ou au contraire les apaiser. Toutefois, l'échange d'informations et les interactions sociales naissant au cours du processus peuvent permettre à des cadets sociaux de s'approprier l'information, d'accéder à la parole, voire de participer au processus de décision.

Par exemple, l'atelier de mise en place d'un comité restreint de suivi du projet au Sénégal a été l'occasion de percevoir quelques enjeux liés à la participation quand les membres des organisations de producteurs ont vivement manifesté leur désaccord à la proposition de coopter les présidents de conseil rural dans ce comité. Ce refus traduit les tensions toujours présentes entre l'administration et la société civile au sujet de l'accès à la terre. La tentative de greffe du droit foncier moderne sur des systèmes coutumiers de gestion est loin d'avoir prise d'autant plus que les vides juridiques et les relations sociales expliquent encore les dérèglements d'affectation précédemment évoqués. Domino se posant, au Sénégal, la question du devenir de la petite exploitation familiale dans ce contexte institutionnel défavorable, la participation au processus de modélisation devient un enjeu en soi.

À La Réunion, la question initiatrice du projet est celle du devenir du foncier agricole soumis aux contraintes précédemment présentées. Mais le secteur agricole n'est pas homogène. La conservation de la sole agricole est un enjeu partagé mais il en existe d'autres qui sont des sources potentielles de tension. Par exemple, doit-on favoriser la concentration des exploitations cannières pour les rendre plus productives et assurer la pérennité des deux dernières usines de transformation? Au contraire, ne doit-on pas accompagner les petits producteurs dont les exploitations ne sont pas viables mais qui composent un

27 Au sens de l'« honneur social » de Weber. 
tissu social important et façonnent en partie le paysage des Hauts? Doit-on favoriser les niches de production dans les zones à potentialité agricole faible ou, à l'inverse, la promotion de l'irrigation pour le développement d'exploitations cannière plus grandes ? Ces enjeux traduisent des luttes de pouvoir essentielles pour le devenir du modèle agricole réunionnais et s'expriment lors du processus de co-construction, notamment dans les simplifications que la modélisation impose.

La capacité à gérer ces tensions est l'un des enjeux principaux de l'implication de la population bénéficiaire dans le projet Domino. L'appropriation des résultats des simulations par les populations cibles est l'un des indicateurs de la réussite du projet, notamment pour évaluer les changements sociaux induits.

\section{Conclusion}

Pourquoi questionner le positionnement des sociologues dans le cadre du projet Domino ? D'abord parce que la démarche de modélisation est participative. Ici la participation n'est pas simplement affichée comme une vitrine répondant aux engouements d'un microcosme scientificopolitique. Elle constitue la colonne vertébrale du dispositif d'accompagnement. Elle se présente comme une sorte de catalyseur pour faire évoluer les rapports au foncier. Elle reconnait les difficultés qui s'insèrent bien souvent dans le court terme, notamment en ce qui concerne la mobilisation de toutes les parties prenantes, la gestion des tensions et du temps. Elle revendique également l'appropriation de la démarche par les acteurs et la construction d'un collectif de réflexion. Cependant, la place du sociologue dans un tel dispositif doit être clarifiée. À la fois observateur du processus en cours d'élaboration dont il analyse la dynamique sociale, et participant à une démarche qu'il alimente par les connaissances qu'il y partage, sa position transcende les catégories traditionnelles de l'action du sociologue.

De plus, en initiant un changement social potentiel en matière d'affectation des terres, que ce soit à La Réunion ou au Sénégal, Domino entre de plain-pied dans une arène politique dont il ne connaît pas nécessairement les tenants et les aboutissants. En travaillant sur le foncier, il intervient dans des jeux de pouvoirs qui bien souvent dépassent la majorité des chercheurs non habitués à intervenir sur de 
tels sujets politiques et sociaux. Pourtant tous les acteurs sont conscients, au moins partiellement, des incohérences et des défauts des systèmes de gestion existants. Il existe une sorte de flou dont la " cohérence " assure une certaine stabilité sociale et dont s'accommodent certains acteurs. Les lacunes portent sur la définition des intérêts des parties prenantes, l'expression de leurs enjeux, la mise en interaction des acteurs dans un espace de recherche-développement à l'écart des lieux traditionnels d'expression des tensions. Elles s'expriment également en matière de légitimation normative (dans les droits modernes ou coutumiers, ou plus simplement dans les valeurs des groupes d'intérêts concernés) de décisions prises par les participants. La démarche d'accompagnement par les principes qu'elle s'est donnée tente de dévoiler puis d'intégrer la pluralité et la complexité de ces enjeux. Face à de tels enjeux sociopolitiques, le sociologue tente d'apporter les clés de lecture permettant aux chercheurs de mieux appréhender le contexte de leur intervention, et aux acteurs de prendre du recul pour mieux saisir la globalité (en termes d'échelles institutionnelles, mais également en termes de définition du système d'acteurs concernés) des interactions pour faciliter le partage de points de vue permettant d'aboutir à l'accord. Ici l'accord n'est pas un consensus " mou ", mais bien le fruit d'une construction collective qui aura veillé - autant que faire se peut - à ce que les positions sociales inégales des partenaires ne perturbent pas l'appropriation des acteurs les moins dotés en privilèges sociaux. La démarche ne vise pas à conforter la position des plus grands "affectataires » et ne s'attache pas non plus à donner plus de pouvoir aux plus vulnérables. Il s'agit d'initier un dialogue fructueux pour chaque catégorie de participant. Cependant, malgré toutes ces précautions, nous ignorons si de tels effets induits ne se produiront pas et qui sont les personnes qui en profiteront. Conscients du problème, nous tentons d'en minimiser les effets éventuels. La démarche invite à agir avec la modélisation, mais au-delà de la modélisation aussi.

La sociologie est une activité scientifique qui trouve « tout naturellement » sa place dans la modélisation d'accompagnement dont la raison d'être consiste en la "présentation », l'exposé de réalités multiples, de mondes pluriels. Ces derniers se présentent, par exemple, sous formes d'acteurs rencontrés, de situations vécues, de sollicitations en tous genres, ou encore d'enjeux variables. La responsabilité du chercheur est alors d'organiser au plus près de sa conscience les liens à même de 
produire un équilibre entre ces mondes. Il tisse des relations sociales et contribue à analyser (verbaliser) le social. Ce faisant, il en devient, lui aussi, acteur, en une posture mettant résolument l'accent sur l'échange et sur l'acceptation des savoirs scientifiques ou pratiques des autres partenaires de la recherche. C'est en clarifiant ce double positionnement dans les projets relatifs à la gestion de systèmes complexes et à ses méthodes d'intervention que le sociologue impliqué pourra asseoir sa légitimité scientifique. On reproche trop souvent aux sciences sociales soit une distance excessive à l'encontre des conséquences sociales de leur recherche, soit des implications militantes éloignées de la neutralité axiologique $^{28}$. Nous suggérons une implication maîtrisée et sûre de son ancrage disciplinaire prenant appui et se nourrissant d'une éthique de responsabilité.

\section{Bibliographie}

Agreste. Mémento agricole : la statistique agricole, Ministère de l'agriculture et de la pêche, Direction de l'agriculture et de la forêt de Ia Réunion, Michel Sinoir (dir.), SaintDenis, La Réunion 2005.

Akrich, Madeleine, Michel Callon et Bruno Latour, « À quoi tient le succès des innovations? 1 ; L'art de l'intéressement 2 : Le choix des porte-parole », Gérer et comprendre. Annales des mines, no 11, 1988, p. 4-17 et no 12, 1988, p. 14-29.

Argent, Robert M. et Rodger B. Grayson, "Modelling shell for participatory assessment and management of natural resources », Environmental modelling and software, vol. 18, n" 6, 2003, p. 541-551.

Bellmann, Klaus, "Towards to a system analytical and modelling approach for integration of ecological, hydrological, economical and social components of disturbed regions ", I.andscape and urban planning, vol. 51, 2000, p. 75-87.

Blandin, Patrick (rapporteur), Vers une évolution durable de l'antbroposystème, "Prospectives Sociétés et environnements », Paris, INSU, 5-6 février 2004, p.115-124.

Boltanski, Luc et Laurent Thévenot, De la justification : les économies de la grandeur, Paris, Gallimard, 1991.

Bourdieu, Pierre, Questions de sociologie, Paris, Minuit, 1980.

Bourdieu, Pierre et Loïc Wacquant, Réponses: pour une antbropologie réflexive, Paris, Seuil, 1992.

28 Bernard Lahire, «Utilité : entre sociologie expérimentale et sociologie sociale », dans Bernard Lahire (dir.), À quoi sert la sociologie ?, Paris, La Découverte / Poche, 2004. 
Collectif ComMod, " La modélisation comme outil d'accompagnement », Nature, sciences et sociétés, vol. 13, n" 2, 2005, p. 165-169.

Daré, William's, Comportements des acteurs dans le jeu et dans la réalité, indépendance ou correspondance? Analyse sociologique de l'utilisation de jeux de rôles en aide à la concertation, Thèse ENGREF, Paris, 2005.

Fraser, Evan D. G., Warren Mabee et Olav Slaymaker, " Mutual vulnerability, mutual dependence, the reflexive relation between human society and the environment ", Global environmental change, n"13, 2003, p. 137-144.

Funtowicz, Silvio O. et Jerry R. Ravetz, «Science for the post-normal age », Futures, vol. $25, n^{\prime \prime} 7,1993$, p. $739-755$.

Giddens, Anthony, The constitution of society, Cambridge, Polity Press, 1986.

Herreros Gilles, Pour une sociologie d'intervention, Paris, Érès, 2002.

Holling, Crawford S., «Engineering resilience versus ecological resilience », dans Peter Schulze (dir.), Engineering within ecological constraints, Washington (D.C.), National Academy, 1996, p. 31-44.

Holling, Crawford S., "Resilience and stability of ecological systems », Annual review of ecology and systematics, $\mathrm{n}^{\circ} 4,1973$, p. 1-23.

Holling, Crawford S., « The resilience of terrestrial ecosystems; local surprise and global change, dans William C. Clark et R. E. Munn (dir.), Sustainable development of the biosphere, Cambridge (U.K.), Cambridge University Press, 1986, p. 292-317.

Holling, Crawford S., "Understanding the complexity of economic, ecological and social systems ", Ecosystems, nं 4, 2001, p. 390-405.

Lahire, Bernard, «Utilité : entre sociologie expérimentale et sociologie sociale », dans Bernard Lahire (dir.), A quoi sert la sociologie?, Paris, La Découverte / Poche, 2004, p. 43-66.

Lavigne Delville, Philippe, Nour-Eddine Sellamna et Marilou Mathieu (dir.), Les enquêtes participatives en débat : ambition, pratiques et enjeux, Paris, Karthala / ICRA/ GRET, 2000.

Le Moigne, Jean-Louis, Le constructivisme : les fondements, vol. 1, Paris, ESF, 1994.

Parker, Dawn C., Steven M. Manson, Marco A. Janssen, Matthew J. Hoffmann et Peter Deadman, "Multi-agent systems for the simulation of land-use and land-cover change: A review "), Annals of the Association of American geographers, vol. 93, n" 2, 2003, p. 314-337.

Schéma Départemental d'Aménagement et de Développement Durable (SDADD), Le diagnostic stratégique et les approches territoriales, Saint-Denis, Département de La Réunion, septembre 2005.

Sirinelli, Jean-François, en collaboration avec Pascal Ory, Les intellectuels en France, de l'affaire Dreyfus à nos jours, Paris, Armand Colin, 2002.

Verret, Michel, Chevilles ouvrières, Paris, Les éditions de l'atelier et Les éditions ouvrières, 1995.

Walker, Brian, Stephen Carpenter, John Anderies, Nick Abel, Graeme Cumming, Marco Janssen, Louis Lebel, Jon Norberg, Garry D. Peterson et Rusty Pritchard, "Resilience management in social-ecosystems : A working hypothesis for a participatory approach », Conservation ecology, vol. 6, $\mathrm{n}^{\circ} 1,2002,14 \mathrm{p}$. 